\title{
Electron tunneling into surface states through an inhomogeneous barrier: asymptotically exact solution of the problem and STM theory
}

\author{
N.V. Khotkevych and Yu.A. Kolesnichenko \\ B. Verkin Institute for Low Temperature Physics and Engineering of the National Academy of Sciences of Ukraine \\ 47 Lenin Ave., Kharkov 61103, Ukraine \\ E-mail: kolesnichenko@ilt.kharkov.ua
}

J.M. van Ruitenbeek

Kamerlingh Onnes Laboratorium, Universiteit Leiden, Postbus 9504, 2300 Leiden, The Netherlands

Received November 12, 2012

\begin{abstract}
We have found an asymptotically exact solution of the Schrödinger equation for electrons tunneling into surface states through an inhomogeneous barrier of large amplitude. Assuming an elliptic dispersion law for the charge carriers the "standing wave" pattern in the conductance of the system resulting from the electron scattering by a single defect in the vicinity of the surface is analyzed.

PACS: 74.55.+v Tunneling phenomena: single particle tunneling and STM;

85.30.Hi Surface barrier, boundary, and point contact devices;

73.50.-h Electronic transport phenomena in thin films.
\end{abstract}

Keywords: STM, electron tunneling, surface states, single defect, electron scattering.

\section{Introduction}

Single surface and subsurface defects result in an interference pattern in images obtained by scanning tunneling microscope (STM) [1] (for review see [2-4]). The nature of a nonmonotonic dependence of the tunnel conductance $G=d I / d V$ on the lateral coordinates, as measured between the STM-tip and the sample, is of the same nature as Friedel oscillations in the electron local density of states (LDOS) in the vicinity of a scatterer [5]. It is the quantum interference between incident electron waves and waves scattered by the defects, leading to the formation of standing waves. Analysis of the oscillatory dependence of the conductance on the STM-tip position relative to the defects gives information about the defect itself and the host metal. Particularly, the depth of the defects below the metal surface [6-9], the magnetic moment of subsurface magnetic clusters [10,11], and contours related to the bulk Fermi surface can be determined [12-16].

Many works deal with STM theory (for reviews see, for example, $[3,17,18])$. One of the widely used models describing STM experiments was proposed by Tersoff and Hamann [19] whose theoretical analysis of the tunnel current is based on Bardeen's formalism [20]. Reference 19 used a model wave function for an isolated STM-tip and an unperturbed wave function of states at the surface of the sample to show that the conductance of the system is proportional to the LDOS of the sample at the tip position. Such an approach reduces the task of the theoretical interpretation of standing wave pattern in the STM images to the calculation of Friedel oscillations in LDOS of surface states (see, for example, [21]).

Another model [22] which can be applied to describe STM experiments [7] is the model of an inhomogeneous barrier of small transparency as part of an infinitly thin interface between two conductors. In framework of this model an asymptotically exact analytical formula for the tunnel conductance can be obtained [22] (in terms of the inverse barrier amplitude), as well as its oscillations due to subsurface defects [7].

In this paper we use the latter model [22] to study the electron tunneling through a small contact into Shockleylike surface states. The scattering of surface electron waves by a single defect incorporated in the sample surface is taken into account. Under assumptions of an elliptic dispersion law for the conduction electrons (i.e., in the approximation of an effective mass tensor which does not depend on momentum), and a small interaction constant 
for scattering of the electrons with the defect, we have found an asymptotically exact expression for the conductance of the system. The relation between the Fermi surface contour and the interference pattern produced in the conductance by the electron scattering is discussed.

The novelty of the present paper is defined by the following: Analytical formulas for the STM conductance describing the electron tunneling into surface states, taking into account their scattering by single defects, have been obtained for arbitrary size of the contact and for an anisotropic (elliptic) Fermi contour. We found exact mathematical relations between shape of the contours in the realspace STM images, their Fourier transform, and the true Fermi contour ellipse.

The organization of this paper is as follows. The model that we use to describe the contact, and the method for obtaining a solution of the Schrödinger equation are described in Sec. 2. In Sec. 3 the differential conductance is found on the basis of a calculation of the probability current density through the contact. Section 4 presents a physical interpretation of the results obtained. In Sec. 5 we conclude by discussing the possibilities for exploiting these theoretical results for interpretation of STM experiments.

\section{Solution of the Schrödinger equation}

The model that we consider is illustrated in Fig. 1. Electrons can tunnel through a small region centered at the point $\mathbf{r}=0$ in an infinitely thin insulating interface at $z=0$ from a half-space $z<0$ (the tip) into a conducting half-space $z>0$. The inhomogeneous potential barrier in the plane $z=0$ we describe by the function $U(\mathbf{r})=$ $=U_{0} f(\rho) \delta(z), \rho=(x, y)$ is a two-dimensional position vector in the plane. The function $f(\rho)$ provides the electron tunneling through a bounded region of range $a$ corresponding to the radius of the contact, and fulfills the conditions,

$$
f(\rho)=\left\{\begin{array}{c}
\sim 1, \rho \lesssim a \\
\rightarrow \infty, \rho \gg a
\end{array}\right.
$$

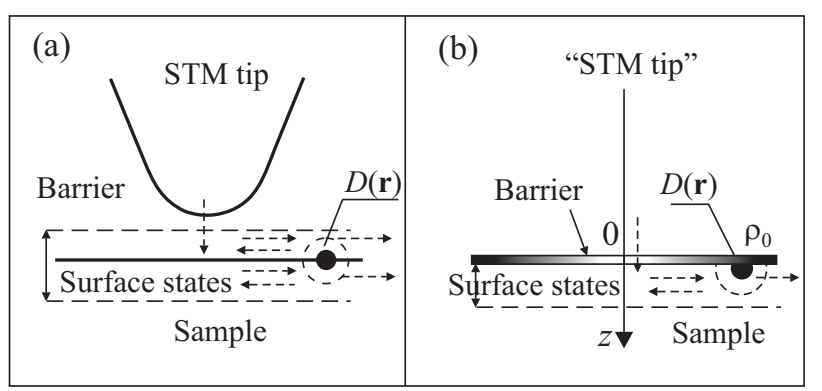

Fig. 1. Schematic representation of a STM experiment. In our model the STM tip (a) has been replaced by an inhomogeneous barrier at $z=0$ (b), which is described by the function $f(\rho)$ (1). Arrows schematically show directions of propagation of electron waves. In the point $\rho_{0}$ a point-like defect describing by potential $D\left(\rho-\rho_{0}, z\right)$ is situated.
In the vicinity of the interface at $z>0$ a single pointlike defect described by a short range potential $D(\mathbf{r})=$ $=g D_{0}\left(\rho-\rho_{0}, z\right)$ is situated, where $g$ is the constant of interaction of the electrons with the defect, and $D_{0}\left(\rho-\rho_{0}, z\right)$ is a function localized within a region $z>0$ of characteristic radius $r_{D}$ around of the point $\mathbf{r}_{0}=\left(\rho_{0},+0\right)$. It satisfies the normalization condition

$$
\int_{-\infty}^{\infty} d \mathbf{r} D_{0}\left(\rho-\rho_{0}, z\right)=1
$$

Surface states at $z>0$ are induced by the surface potential $V_{s}(z)$. In order to obtain an analytical solution of the Schrödinger equation and calculate the electric current in what follows we use an anisotropic dispersion law $\varepsilon(\mathbf{k})$ for the charge carriers in both conducting half-spaces, where $\mathbf{k}$ is the electron wave vector, and for concreteness we take it to be elliptical. The wave function $\psi(\mathbf{r})$ then satisfies the Schrödinger equation

$$
\begin{aligned}
& \frac{\hbar^{2}}{2}\left(\frac{\partial^{2}}{m_{x} \partial x^{2}}+\frac{\partial^{2}}{m_{y} \partial y^{2}}+\frac{\partial^{2}}{m_{z} \partial z^{2}}\right) \psi(\mathbf{r})+ \\
& +\left[\varepsilon-U(\mathbf{r})-D(\mathbf{r})-V_{s}(z)\right] \psi(\mathbf{r})=0 .
\end{aligned}
$$

Here, $\varepsilon$ is the electron energy, and $m_{k}>0$ are the principal values of effective mass tensor.

Let us search for solutions of Eq. (3) corresponding to electron tunneling from the surface states at $z>0$ into the bulk tip states in the half-space $z<0$. Hereafter we follow the procedure for the finding the electron wave functions in the limits $U_{0} \rightarrow \infty$ and $g \rightarrow 0$ that was proposed in Refs. 7,22 and search for the wave function $\psi(\mathbf{r})$ as an expansion into a series:

$$
\psi(\mathbf{r})=\psi_{0}(\mathbf{r})+\frac{1}{U_{0}} \varphi_{0}(\mathbf{r})+g \psi_{1}(\mathbf{r})+\frac{g}{U_{0}} \varphi_{1}(\mathbf{r})+\ldots
$$

The functions $\psi_{0,1}(\mathbf{r})$ of the zeroth order approximation in $1 / U_{0}$ satisfy the boundary condition at the interface $z=0$

$$
\psi_{0,1}(\rho, 0)=0,
$$

while the functions $\psi_{0,1}(\mathbf{r})$ satisfy the continuity condition

$$
\varphi_{0,1}(\rho,+0)=\varphi_{0,1}(\rho,-0)
$$

and the condition for jump in the derivative at $z=0$, which for large $U_{0}$ reduces to the condition $[7,22]$

$$
\left.\mp \frac{\partial}{\partial z}\left\{\begin{array}{l}
\psi_{0}(\mathbf{r}) \\
\psi_{1}(\mathbf{r})
\end{array}\right\}\right|_{z=\mp 0}=\left.\frac{2 m^{*}}{\hbar^{2}} f(\boldsymbol{\rho})\left\{\begin{array}{l}
\varphi_{0}(\mathbf{r}) \\
\varphi_{1}(\mathbf{r})
\end{array}\right\}\right|_{z=0} .
$$

In leading approximation in the constant $g$ the functions $\psi_{1}(\mathbf{r})$ and $\varphi_{1}(\mathbf{r})$ can be written as 


$$
\left\{\begin{array}{l}
\psi_{1}(\mathbf{r}) \\
\varphi_{1}(\mathbf{r})
\end{array}\right\}=\int_{-\infty}^{\infty} d \mathbf{r}^{\prime} D_{0}\left(\boldsymbol{\rho}^{\prime}-\rho_{0}, z^{\prime}\right) G_{0}^{+}\left(\mathbf{r}, \mathbf{r}^{\prime} ; \varepsilon\right)\left\{\begin{array}{l}
\psi_{0}\left(\mathbf{r}^{\prime}\right) \\
\varphi_{0}\left(\mathbf{r}^{\prime}\right)
\end{array}\right\}
$$

where $G_{0}^{+}\left(\mathbf{r}, \mathbf{r}^{\prime} ; \varepsilon\right)$ is the retarded Green's function for the unperturbed surface states in the field of the potential $V_{\text {im }}(z)$ near an impenetrable interface.

The wave function in the zeroth approximation $\psi_{0}(\mathbf{r})$, as well as the Green's function $G_{0}^{+}\left(\mathbf{r}, \mathbf{r}^{\prime} ; \varepsilon\right)$, can be easily found. For the wave function we have,

$$
\psi_{0}(\mathbf{r})=\frac{1}{\sqrt{L_{x} L_{y}}} \mathrm{e}^{i \kappa \rho} \chi_{0}(z)
$$

In Eqs. (9) and (12) below, $\boldsymbol{\kappa}$ is a two-dimensional electron wave vector parallel to the interface, $L_{x}, L_{y}$ are the sizes of the interface $\left(L_{x, y} \rightarrow \infty\right)$, and $\chi_{0}(z)$ is the solution of the equation

$$
\frac{\hbar^{2}}{2 m_{z}} \frac{\partial^{2} \chi_{0}(z)}{\partial z^{2}}+\left(\varepsilon_{0}-V_{s}(z)\right) \chi_{0}(z)=0, \quad z \geqslant 0
$$

subject to boundary conditions and normalization,

$$
\chi_{0}(0)=0, \quad \chi_{0}(z \rightarrow \infty) \rightarrow 0, \int_{0}^{\infty} d z\left|\chi_{0}(z)\right|^{2}=1 .
$$

The electron energy corresponding to the state (9) is $\varepsilon=\varepsilon_{\| \mid}(\kappa)+\varepsilon_{0}$, where $\varepsilon_{\| \mid}(\kappa)=\hbar^{2} \kappa_{x}^{2} / 2 m_{x}+\hbar^{2} \kappa_{y}^{2} / 2 m_{y}$ is the energy of the electron motion in a plane $z=$ const, and $\varepsilon_{0}$ is the electron energy level in the potential well formed by the potential $V_{\mathrm{im}}(z)$ and the infinite wall at $z=0$. We will assume that there is ony one filled quantum level below the Fermi level $\varepsilon_{\mathrm{F}}$.

The function $\psi_{0}(\mathbf{r})$ in Eq. (9) is the wave function for the surface states in an ideal conducting half-space when neglecting the finite value of a work function. The function $\psi_{1}(\mathbf{r})$ describes the perturbation of $\psi_{0}(\mathbf{r})$ by electron scattering on the defect.

The Green's function of the surface states is given by

$$
G_{0}^{+}\left(\mathbf{r}, \mathbf{r}^{\prime} ; \varepsilon\right)=\frac{i \sqrt{m_{x} m_{y}}}{2 \hbar^{2}} \chi_{0}(z) \chi_{0}^{*}\left(z^{\prime}\right) H_{0}^{(1)}\left(\Gamma\left(\varepsilon-\varepsilon_{0}, \rho, \rho^{\prime}\right)\right),
$$

where $H_{0}^{(1)}$ is the Hankel function, with

$$
\Gamma\left(\varepsilon, \rho, \rho^{\prime}\right)=\frac{1}{\hbar} \sqrt{2 \varepsilon\left[m_{x}\left(x-x^{\prime}\right)^{2}+m_{y}\left(y-y^{\prime}\right)^{2}\right]} .
$$

The wave function for the electrons transmitted through the barrier can be found by the method described in Refs. 7,22,

$$
\begin{aligned}
& \varphi(\mathbf{r})=\varphi_{0}(\mathbf{r})+g \varphi_{1}(\mathbf{r})=\frac{\hbar^{2} \chi_{0}^{\prime}(+0)}{8 \pi^{2} m_{z} U_{0} \sqrt{L_{x} L_{y}}} \int_{-\infty}^{\infty} d \rho^{\prime} \frac{\mathrm{e}^{i \mathbf{\kappa} \boldsymbol{\rho}^{\prime}}}{f\left(\boldsymbol{\rho}^{\prime}\right)} \int_{-\infty}^{\infty} d \mathbf{k}^{\prime} \mathrm{e}^{i \mathbf{\kappa}^{\prime}\left(\boldsymbol{\rho}-\boldsymbol{\rho}^{\prime}\right)} \mathrm{e}^{-i k_{z}^{\prime}|z|} \times \\
& \quad \times\left\{1+\frac{i g \sqrt{m_{x} m_{y}}}{2 \hbar^{2}} \int_{-\infty}^{\infty} d \mathbf{r}^{\prime \prime} D_{0}\left(\rho^{\prime \prime}-\rho_{0}, z^{\prime \prime}\right)\left|\chi_{0}\left(z^{\prime \prime}\right)\right|^{2} \mathrm{e}^{i \mathbf{k} \boldsymbol{\rho}^{\prime \prime}} H_{0}^{(1)}\left(\Gamma\left(\varepsilon-\varepsilon_{0}, \boldsymbol{\rho}^{\prime}, \boldsymbol{\rho}^{\prime \prime}\right)\right)\right\} .
\end{aligned}
$$

\section{Standing wave pattern in the point contact conductance}

For small transparency of the potential barrier the profile of the electric potential can be approximated by a step function $V \Theta(-z)$. We take the temperature $T=0$ and $\mathrm{eV}<0$, and consider only the electron flow from the surface states at $z>0$ into bulk tip states at $z<0$ (Fig. 2). Obviously, in the case of a small applied bias $|e V| \ll \varepsilon_{F}$, which we consider in this work, the conductance $G=I(0) / V$ does not depend on the current direction. In the general case of non-Ohmic behavior the currentvoltage characteristic $I(V)$ is not symmetric relative to $V=0$ and the differential conductance $G(V)=d I / d V$ must be calculated for each voltage sign separately, as was done for a small contact on the surface of a thin film in Ref. 23. The conductance of the system can be found by integration the total probability flux $J$ over an arbitrary plane $z=$ const, $z<0$

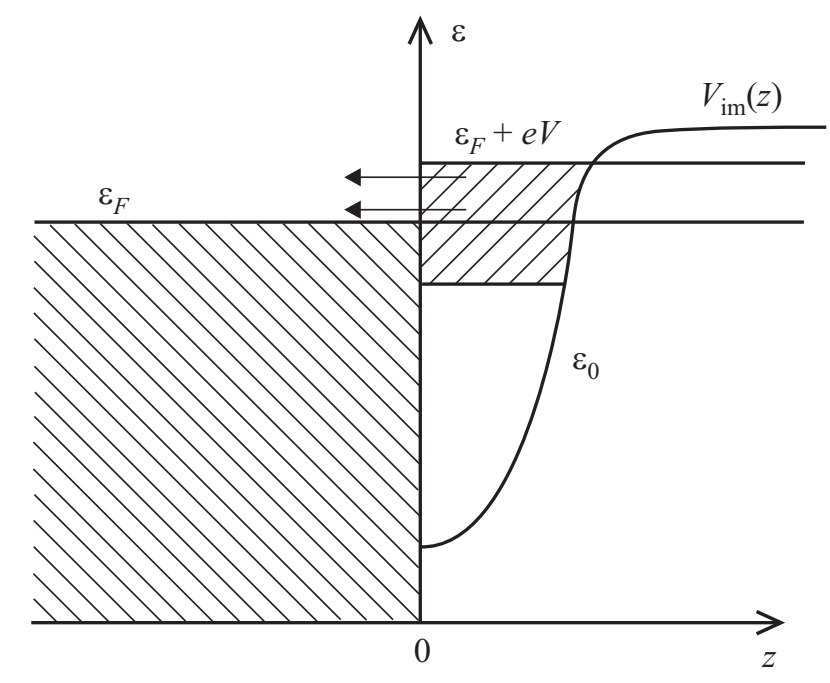

Fig. 2. Illustration of the occupied energy bands. Arrows show the direction of electron tunneling from surface states into bulk states. 


$$
G=-\frac{e^{2} \hbar L_{x} L_{y}}{2 \pi^{2} m_{z}} \int_{-\infty}^{\infty} d \kappa \int_{-\infty}^{\infty} d \rho \operatorname{Im}\left[\varphi^{*}(\rho, z) \frac{\partial}{\partial z} \varphi(\rho, z)\right] \frac{\partial f_{F}(\varepsilon)}{\partial \varepsilon} .
$$

It is natural that the result of integration in Eq. (15) does not depend on $z$. Substituting the wave function $\varphi(\rho, z)$ (14), into Eq.(15) we find after some integration

$$
\begin{gathered}
G=\frac{e^{2} \varepsilon_{F}^{3 / 2} m_{x} m_{y}\left|\chi_{0}^{\prime}(+0)\right|^{2}}{2^{3 / 2}\left(m_{z}\right)^{5 / 2} \pi^{2} U_{0}^{2}} \int_{-\infty}^{\infty} \int_{-\infty}^{\infty} \frac{d \rho^{\prime}}{f\left(\rho^{\prime}\right)} \frac{d \rho^{\prime \prime}}{f\left(\rho^{\prime \prime}\right)} \frac{j_{1}\left(\Gamma\left(\varepsilon_{F}, \rho^{\prime}, \rho^{\prime \prime}\right)\right)}{\Gamma\left(\varepsilon_{F}, \rho^{\prime}, \rho^{\prime \prime}\right)}\left[J_{0}\left(\Gamma\left(\varepsilon_{F}-\varepsilon_{0}, \rho^{\prime}, \rho^{\prime \prime}\right)\right)-\right. \\
\left.-\frac{g \sqrt{m_{x} m_{y}}}{\hbar^{2}} \int_{-\infty}^{\infty} d \rho^{\prime \prime \prime} \int_{-\infty}^{\infty} d z^{\prime \prime \prime} D_{0}\left(\rho^{\prime \prime \prime}-\rho_{0}, z^{\prime \prime \prime}\right)\left|\chi_{0}\left(z^{\prime \prime \prime}\right)\right|^{2} J_{0}\left(\Gamma\left(\varepsilon_{F}-\varepsilon_{0}, \rho^{\prime}, \rho^{\prime \prime \prime}\right)\right) Y_{0}\left(\Gamma\left(\varepsilon_{F}-\varepsilon_{0}, \rho^{\prime}, \rho^{\prime \prime \prime}\right)\right)\right],
\end{gathered}
$$

where $J_{0}(x)$ and $Y_{0}(x)$ are Bessel functions of the first and the second kind. Further calculations require explicit expressions for the functions $f(\rho)$ and $D_{0}\left(\rho-\rho_{0}, z\right)$. The integral formula (16) can be simplified for small contacts (contact radius $a \rightarrow 0$ ) and for a short range potential $\left(r_{D} \rightarrow 0\right)$. In these limits all functions in the integrals, except $f(\rho)$ and $D_{0}\left(\rho-\rho_{0}, z\right)$, can be taken in the points $\rho^{\prime}=\rho^{\prime \prime}=0, \rho^{\prime \prime \prime}=\rho_{0}$ and Eq.(16) is reduced to

$$
G\left(\rho_{0}\right)=\frac{2 \pi e^{2}}{\hbar} T\left(S_{\text {eff }}\right) \rho_{3 D}^{(0)} \rho_{2 D}\left(\rho_{0}\right) .
$$

Here

$$
\rho_{2 D}\left(\rho_{0}\right)=\rho_{2 D}^{(0)}\left[1-\frac{\tilde{g} \sqrt{m_{x} m_{y}}}{\hbar^{2}} J_{0}\left(h\left(\mathbf{n}_{0}\right) \rho_{0}\right) Y_{0}\left(h\left(\mathbf{n}_{0}\right) \rho_{0}\right)\right],
$$

$$
\rho_{2 D}^{(0)}=\frac{\sqrt{m_{x} m_{y}}}{\pi \hbar^{2}}, \quad \rho_{3 D}^{(0)}=\frac{\sqrt{2 \varepsilon_{F} m_{x} m_{y} m_{z}}}{(\pi \hbar)^{3}},
$$

are the densities of states (for two spin directions) for defect-free two-dimensional (2D) and three-dimensional (3D) systems, $\rho_{2 D}^{(0)}$ and $\rho_{3 D}^{(0)}$, and local density of surface states scattered by single defect, $\rho_{2 D}\left(\rho_{0}\right)$. In these expressions,

$$
h\left(\mathbf{n}_{0}\right)=\frac{1}{\hbar} \sqrt{2\left(\varepsilon_{F}-\varepsilon_{0}\right)\left(m_{x} \cos ^{2} \vartheta_{0}+m_{y} \sin ^{2} \vartheta_{0}\right)},
$$

$\mathbf{n}_{0}=\left(\cos \vartheta_{0}, \sin \vartheta_{0}\right)$ defines the angle between the point contact and the position of the defect $\rho_{0}=\rho_{0}\left(\cos \vartheta_{0}, \sin \vartheta_{0}\right)$, and the coefficient $T\left(S_{\text {eff }}\right)$, which depends on the effective area of the contact, plays the role of the tunneling matrix element,

$$
T\left(S_{\text {eff }}\right)=\frac{\pi \varepsilon_{F} \hbar^{6}}{24 m_{z}^{3} U_{0}^{2}}\left|\chi_{0}^{\prime}(+0)\right|^{2} S_{\text {eff }}^{2},
$$

$$
S_{\text {eff }}=\int_{-\infty}^{\infty} \frac{d \rho}{f(\rho)}
$$

and

$$
\tilde{g}=g \int_{-\infty}^{\infty} d \rho \int_{-\infty}^{\infty} d z D_{0}\left(\rho-\rho_{0}, z\right)\left|\chi_{0}(z)\right|^{2}
$$

is the effective constant of interaction of electrons belonging to the surface states with the defect at $\rho_{0}=\rho_{0}\left(\cos \vartheta_{0}, \sin \vartheta_{0}\right)$. Note that the limit Eq. (17) can be used for describing experimental data with satisfactory accuracy, as long as $a$ and $r_{D}$ are smaller than the Fermi wave length $\lambda_{F}$.

Figure 3 illustrates the standing wave pattern in the conductance $G\left(\rho_{0}\right)$, Eq. (17), around the defect. Maxima and minima in Fig. 3 form curves of constant phase of oscillatory functions in Eq. (17), $2 h\left(\vartheta_{0}\right) \rho_{0}=$ const, and the visible contours are defined by the function

$$
\rho_{0}\left(\vartheta_{0}\right)=\frac{\text { const }}{2 h\left(\vartheta_{0}\right)} \text {. }
$$

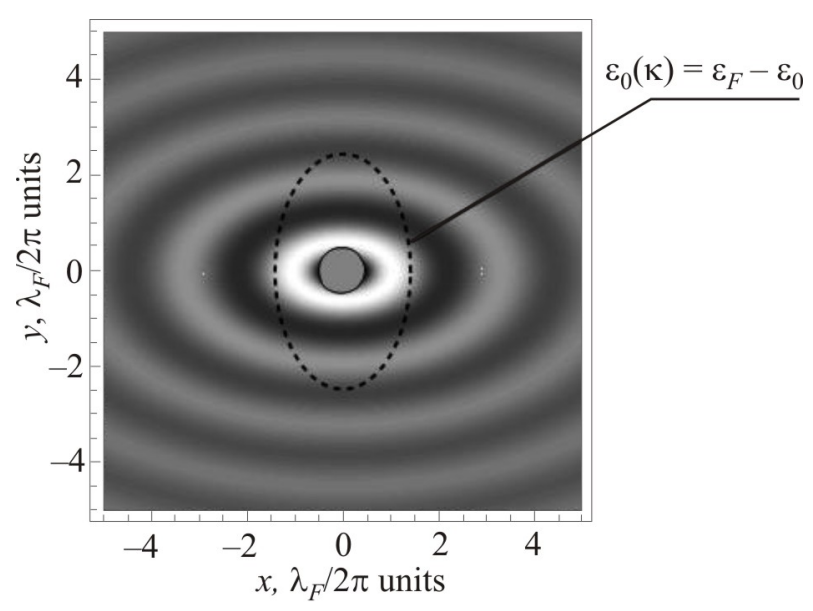

Fig. 3. Standing wave pattern in the conductance $G$ as described by Eq. (17). The dashed ellipse is the Fermi contour in momentum space. The center of the defect is placed in the point $\rho=0$, and $\lambda_{F} / 2 \pi=\hbar / \sqrt{2 m_{x}\left(\varepsilon_{F}-\varepsilon_{0}\right)}$, with $m_{y}=3 m_{x}$. 


\section{Discussion}

Thus, in the framework of the model illustrated in Fig. 1(b) we have obtained a formula for the conductance, that is asymptotically exact in the limit of small transparency of the barrier between conductors, for an inhomogeneous tunnel barrier of arbitrary form allowing electron tunneling from the surface states into the bulk states in the other conductor (16). For the sake of clarity, in discussing our results we make reference to the simplified formula for the conductance, Eq.(17).

The Fermi contour of the surface states for an elliptic dispersion law can be written in polar coordinates, with the center in the center of the ellipse, as

$$
k(\phi)=\frac{\sqrt{2\left(\varepsilon_{F}-\varepsilon_{0}\right) m_{x} m_{y}}}{\hbar \sqrt{\left(m_{y} \cos ^{2} \phi+m_{x} \sin ^{2} \phi\right)}},
$$

$\mathbf{k}=k(\cos \phi, \sin \phi)$. The auxiliary function $h(\phi)$ is the function that measures the distance from the tangent line at the point $k(\phi)$ on the ellipse to the origin $\mathbf{k}=0$ [24], i.e. in $k$-space $h(\phi)$ is the projection of $\mathbf{k}(\phi)$ to the normal $\mathbf{n}(\phi)$ to the curve $k(\phi)$ in the point defined by the angle $\phi$,

$$
\begin{gathered}
\mathbf{n}(\phi)=\frac{m_{y} \cos \phi \mathbf{i}+m_{x} \sin \phi \mathbf{j}}{\sqrt{\left(m_{y} \cos \phi\right)^{2}+\left(m_{x} \sin \phi\right)^{2}}} \\
h(\phi)=\mathbf{k}(\phi) \mathbf{n}(\phi)=\frac{\sqrt{2\left(\varepsilon_{F}-\varepsilon_{0}\right) m_{x} m_{y}\left[\left(m_{y} \cos ^{2} \phi+m_{x} \sin ^{2} \phi\right)\right]}}{\hbar \sqrt{\left(m_{y} \cos \phi\right)^{2}+\left(m_{x} \sin \phi\right)^{2}}} .
\end{gathered}
$$

If instead of the angle $\phi$ we choose as a variable in the functions $\mathbf{n}(\phi)$ (26) and $h(\phi)$ (27) the angle $\vartheta$ between the normal vector $\mathbf{n}(\vartheta)=(\cos \vartheta, \sin \vartheta)$ and the $x$-axis (the change of variable is clear from Eq. (26)), the Eq. (27) for the auxiliary function $h(\vartheta)$ takes the same form as Eq. (20). Naturally, at $m_{x}=m_{y}$ the auxiliary function (27) coincides with the contour curve (25) that is the circle. For our model of the Fermi surface the function $\rho_{0}\left(\vartheta_{0}\right)$ (24) gives ellipses rotated relative to the true Fermi contour (25) by an angle $\pi / 2$ (see Fig. 3) This observation is a geometrical property of the ellipse and is not satisfied for any curve.

At large distances from the defect $h \rho_{0} \gg 1$ the oscillatory part $G_{\text {osc }}$ of the conductance (17) takes the simple form

$$
\frac{G_{\text {osc }}}{G_{0 S}} \simeq \frac{\tilde{g} \sqrt{m_{x} m_{y}}}{\pi \hbar^{2}} \frac{\cos \left(2 h\left(\mathbf{n}_{0}\right) \rho_{0}\right)}{h\left(\mathbf{n}_{0}\right) \rho_{0}}, \quad h \rho_{0} \gg 1, \quad k_{F} a \ll 1 .
$$

The Fourier transform of Eq. (28) given by,

$$
\begin{gathered}
F(\mathbf{k})=\int_{-\infty}^{\infty} d \rho_{0} \mathrm{e}^{i \mathbf{k} \rho_{0}} \frac{G_{\mathrm{OSc}}\left(\rho_{0}\right)}{G_{0 S}}= \\
=\frac{\pi \tilde{g}}{\left(\varepsilon_{F}-\varepsilon_{0}\right)} \frac{\theta\left(\frac{\hbar^{2} k_{x}^{2}}{2 m_{x}\left(\varepsilon_{F}-\varepsilon_{0}\right)}+\frac{\hbar^{2} k_{y}^{2}}{2 m_{y}\left(\varepsilon_{F}-\varepsilon_{0}\right)}-4\right)}{\sqrt{\frac{\hbar^{2} k_{x}^{2}}{2 m_{x}\left(\varepsilon_{F}-\varepsilon_{0}\right)}+\frac{\hbar^{2} k_{y}^{2}}{2 m_{y}\left(\varepsilon_{F}-\varepsilon_{0}\right)}-4}}
\end{gathered}
$$

directly gives us the true Fermi contour, of double the original size.

\section{Conclusion}

In summary, we have investigated the electron tunneling from surface states into bulk states through an inhomogeneous barrier of arbitrary form. An asymptotically exact formula for the conductance of the system is obtained for an anisotropic quadratic dispersion law of charge carriers. The influence of electron scattering by a single point-like defect at the surface has been taken into account. We believe that the results obtained are suitable for describing STM experiments. In the framework of our model we have found explicit geometrical relations between the characteristics of the Fermi surface (principal values of the effective mass tensor; the Fermi energy) and curves of constant phase (for example, curves of maxima or minima of the oscillation amplitude) in the standing wave pattern in the real space STM images. Our results authenticate that the real space image does not give us the true Fermi contours, while the Fourier transform makes it possible to reconstruct the Fermi contour of the surface states correctly.

1. M.F. Crommie, C.P. Lutz, and D.M. Eigler, Nature 363, 524 (1993); M.F. Crommie, C.P. Lutz, and D.M. Eigler, Science 262, 218 (1993).

2. L. Petersen, Ph. Hofmann, E.W. Plummer, and F. Besenbacher, J. Electr. Spectr. Relat. Phenom. 109, 97 (2000).

3. Ye.S. Avotina, Yu.A. Kolesnichenko, and J.M. van Ruitenbeek, Fiz. Nizk. Temp. 36, 1066 (2010) [Low Temp. Phys. 36, 849 (2010)].

4. L. Simon, C. Bena, F. Vonau, M. Cranney, and D Aube, J. Phys. D: Appl. Phys. 44, 464010 (2011).

5. J. Friedel, Nuovo Cimento 7, 287 (1958).

6. K. Kobayashi, Phys. Rev. B 5417029 (1996).

7. Ye.S. Avotina, Yu.A. Kolesnichenko, A.N. Omelyanchouk, A.F. Otte, and J.M. van Ruitenbeek, Phys. Rev. B 71, 115430 (2005).

8. Ye.S. Avotina, Yu.A. Kolesnichenko, A.F. Otte, and J.M. van Ruitenbeek, Phys. Rev. B 75, 125411 (2007).

9. N.V. Khotkevych, Yu.A. Kolesnichenko, and J.M. van Ruitenbeek, Fiz. Nizk. Temp. 37, 64 (2011) [Low Temp. Phys. 37, 53 (2011)].

10. Ye.S. Avotina, Yu.A. Kolesnichenko, and J.M. van Ruitenbeek, Phys. Rev. B 80, 115333 (2009). 
11. O.O. Brovko and V.S. Stepanyuk, Phys. Status Solidi B 247, 1161 (2010).

12. Ye.S. Avotina, Yu.A. Kolesnichenko, A.F. Otte, and J.M. van Ruitenbeek, Phys. Rev. B 74, 085411 (2006).

13. Ye.S. Avotina, Yu.A. Kolesnichenko, S.B. Roobol, and J.M. van Ruitenbeek, Fiz. Nizk. Temp. 34, 268 (2008) [Low Temp. Phys. 34, 207 (2008)].

14. A. Weismann, M. Wenderoth, S. Lounis, P. Zahn, N. Quaas, R.G. Ulbrich, P.H. Dederichs, and S. Blügel, Science 323, 1190 (2009).

15. S. Lounis, P. Zahn, A. Weismann, M. Wenderoth, R.G. Ulbrich, I. Mertig, P.H. Dederichs, and S. Blügel, Phys. Rev. $B$ 83, 035427 (2011).

16. C. Pauly, M. Grob, M. Pezzotta, M. Pratzer, and M. Morgenstern, Phys. Rev. B 81, 125446 (2010).

17. W.A. Hofer, A.S. Foster, and A.L. Shluger, Rev. Mod. Phys. 75, 1287 (2003).
18. J.M. Blanco, F. Flores, and Rubén Pérez, Progr. Surface Science 81, 403 (2006).

19. J. Tersoff and D. Hamann, Phys. Rev. Lett. 50, 1998 (1983); Phys. Rev. B 31, 805 (1985).

20. J. Bardeen, Phys. Rev. Lett. 6, 57 (1961).

21. L.C. Davis, M.P. Everson, and R.C. Jaklevic, Phys. Rev. B 43, 3821 (1991).

22. I.O. Kulik, Yu.N. Mitsai, and A.N. Omel'yanchuk, Sov. Phys. JETP 39, 514 (1974).

23. N.V. Khotkevych, Yu.A. Kolesnichenko and J.M. van Ruitenbeek, Fiz. Nizk. Temp. 38, 644 (2012) [Low Temp. Phys. 38, 503 (2012)].

24. H.W. Guggenheimer, Differential Geometry, General Publishing Company (1977). 